\title{
CRITICA A LOS PRINCIPIOS FUNDAMENTALES DE LA FISICA: EL PROBLEMA DE LA PARTICULA ELEMENTAL
}

\author{
Oscar S. Monroy Cárdenas ${ }^{\mathrm{a}}$ \\ ${ }^{a}$ Departamento de Física del Nuclear Atómica y Molecular. Facultad de Ciencias Físicas, Universidad Nacional Mayor de \\ San Marcos Apartado postal 14 -0149, Lima 14 - Perú
}

\begin{abstract}
Resumen
Una revisión del concepto de partícula elemental indivisible, como constituyente básico de la materia, nos conduce a contradicciones con los principios de las teorías físicas convencionales. Este concepto primitivo sería la causa del retraso, entorpecimiento y desviación de la labor fundamentalista del físico en la Tierra.
\end{abstract}

Palabras claves: Planck, Einstein, cuántica, Heisenberg.

\begin{abstract}
When reviewing the concept of indivisible elementary particle, as a basic component of matter, we face contradictions with the principles of the conventional physics theories. The primary concept of elementary particle may be the cause of the confusion and hindering of the fundamental tasks of the physicist on.
\end{abstract}

Keywords: Planck, Einstein, Quantum, Heisenberg.

\section{Introducción.}

En principio, nadie sabe verdaderamente qué es una partícula elemental. Este desconocimiento es natural, si nos damos cuenta que las partículas elementales son producto del pensamiento puro, iniciado por primitivos pensadores griegos como Leucipo y Demócrito, quienes postularon que la materia era discontinua y que la última porción de ella, llamada átomo, debía ser indivisible y en consecuencia invisible. Lamentablemente la perniciosa idea de partícula fundamental indivisible resurgió con la equivocada interpretación de los resultados del experimento de Thomson, el cual generó el desarrollo del atomismo en Europa hasta llegar a las teorías físicas y tecnologías que hoy observamos. Sin embargo, si nos basamos en el argumento lógico y racional de que las proposiciones de los seres humanos acerca del funcionamiento de la naturaleza (llamados principios físicos) deben comprobarse en el propio laboratorio de la naturaleza, y no en un laboratorio artificial diseñado a partir de ideas personales, estaríamos en un grave error conceptual lo cual implica que todo el esfuerzo intelectual realizado hasta hoy en desarrollar teorías, diseñar experimentos y tecnologías representa una desviación del camino lógico que debe seguir todo verdadero científico, y en el futuro, ¡todos los seres humanos! El físico tradicional europeo cometió el grave error de no seguir el ejemplo de Galileo Galilei: estudiar el cosmos en base a la observación directa, y no seguir insistiendo en desarrollar conceptos nebulosos de varios miles de años de antigüedad. Por esta razón se ha incubado innecesariamente tanto artificialismo en la física experimental y tanta complejidad matemática en la física teórica contemporánea. Por tanto, debido a su precaria observación e interpretación de la naturaleza, el 
físico tradicional es quizás el científico que más se engaña a si mismo. Nunca sabe realmente lo que hace ni lo que dice. Sus logros son engañosos tanto en las teorías como en los experimentos. Siempre colapsará en un círculo vicioso invadido por el desconcierto. El propósito de este trabajo es proporcionar observaciones útiles que contribuyan a despertar la conciencia de los verdaderos físicos del mundo de modo que les motive a reflexionar con humildad sobre estas cuestiones tan fundamentales para que puedan decidir objetivamente si continúan o renuncian a considerar la idea de la partícula elemental en nuevas teorías físicas, así como en experimentos diseñados a partir de dichas teorías.

\section{El fracaso del modelo de la partícula puntual.}

2.1. Durante muchos años se imaginaron a los átomos como esferas sólidas e indestructibles de los que estaban constituidos todos los objetos materiales. A raíz de los experimentos de Thomson y de Rutherford el concepto primitivo del átomo cambió, hasta imaginarlo como un objeto compuesto de electrones que orbitan alrededor de un núcleo que a su vez estaba compuesto de protones y neutrones muy próximos entre si. Posteriormente con la invención de los aceleradores de partículas y la consecuente interpretación clásica de colisiones de alta energía condujo a la idea de que los protones y neutrones (así como otras partículas subatómicas obtenidas) estaban constituidos de dos o tres partículas llamadas quarks. En la actualidad, los físicos tradicionales tienen la creencia de que toda la materia del Universo está hecha esencialmente de electrones y quarks los cuales son aceptados como partículas elementales. El modelo de los electrones y de los quarks como esferas indeformables e indestructibles presentó graves dificultades. Imaginemos que un quark y un electrón chocan como dos bolas de billar, entonces para que el quark (o el electrón) no se deforme la fuerza del golpe debería transmitirse simultáneamente e instantáneamente a todas sus partes. Pero uno de los principios de la teoría de la relatividad prohíbe que ninguna influencia viaje más rápido que la luz. En la Fig.1 mostrada esto significaría que la fuerza del impacto en el punto $A$ de un quark tardará en llegar a un punto opuesto B un intervalo de tiempo igual a $\mathrm{L} / \mathrm{v}$, donde la rapidez $\mathrm{v}<\mathrm{c}$ (rapidez de la luz en el vacío). Entonces se deduce que el punto de impacto A se moverá antes que el punto más alejado $\mathrm{B}$, y esto implica que la bola se deforme, lo cual contradice la hipótesis de que el quark (o el electrón) debería ser indeformable. ¡A menos que existan dentro del quark fuerzas recuperadoras elásticas inimaginables!

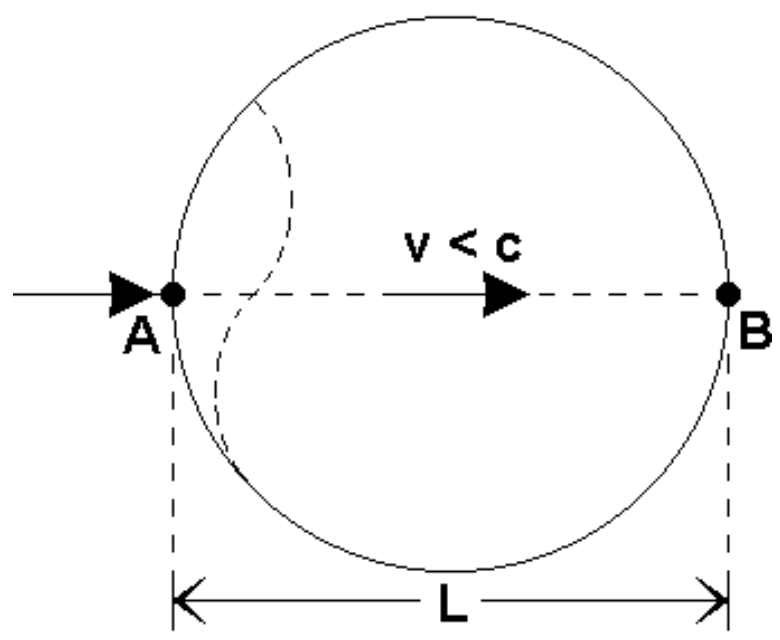

Fig. 1. Deformación de una partícula elemental (líneas discontinuas). Esto se debe a que el punto A se mueve antes que el punto B, mientras que la influencia del impacto viaja con rapidez $\mathrm{v}<\mathrm{c}$.

2.2. Ahora supóngase que los quarks y los electrones son indestructibles porque no tienen estructura interna, o sea, son partículas puntuales que no ocupan ningún volumen. Esta idea también produce graves dificultades. Por ejemplo, imaginemos primero a un quark como una esfera finita con su carga eléctrica distribuida uniformemente. Debido a que la carga eléctrica es del mismo signo, es claro que a medida que se le comprime a la esfera se gastará más y más energía, y la esfera irá acumulando más y más de esta energía en su volumen. Pero sabemos que la energía eléctrica (E) de la esfera es inversamente proporcional a su radio (r), o sea:

$$
\mathrm{E}=\frac{\mathrm{k}}{\mathrm{r}},
$$

Donde $\mathrm{k}$ es una constante. Siguiendo el razonamiento anterior, a medida que el radio de la esfera $r \rightarrow 0$ (ver la figura 2) el quark se aproximará a una partícula puntual, y cuando 
$\mathrm{r}=0$, será una partícula puntual. Pero en este caso de la Ec.(1) se obtiene:

$$
\mathrm{E}=\infty \quad \text { (energía infinita) }
$$

Por otro lado, según la ecuación de Einstein, la energía tiene masa, es decir:

$$
\mathrm{E}=\mathrm{mc}^{2},
$$

Donde $\mathrm{m}$ es la masa del quark y c la rapidez de la luz en el vacío. En consecuencia del resultado (2) y de la Ec.(3) se deduce:

$\mathrm{m}=\infty \quad$ (masa infinita)

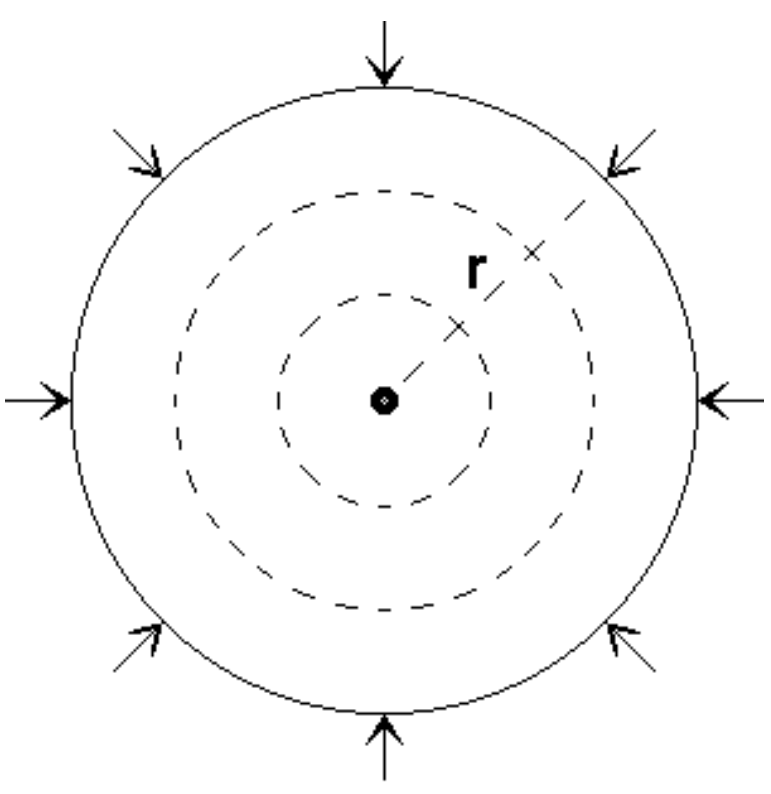

Fig. 2. A medida que se comprime una partícula hasta convertirla en puntual, su energía crece hacia el infinito.

El resultado (4) es evidentemente absurdo porque nos dice que el quark, y todas las partículas subatómicas, deben ser infinitamente "pesadas". Sin embargo esto emerge de combinar las fórmulas (1) y (3) las cuales son supuestamente reconocidas como correctas. Por tanto, la conclusión es que hay algo muy equivocado inherente a todas las teorías de la materia subatómica, iy ese algo debe ser el modelo atómico de la materia!

2.3. El principio de incertidumbre de Heisenberg aplicado a la energía $(\Delta \mathrm{E})$ - tiempo $(\Delta \mathrm{t})$, se expresa por

$$
\Delta \mathrm{E} \Delta \mathrm{t} \geqslant \mathrm{h},
$$

Donde h es la constante de Planck. Este principio plantea la posibilidad de obtener energía aparentemente de la nada, y esto implica la violación de la ley de conservación de la energía para crear materia en las regiones microscópicas del espacio. Sin embargo este hecho no se percibiría a escala humana. Se piensa que este fenómeno ocurre en el enorme espacio vacío que hay en el interior de los átomos donde la energía puede aparecer aparentemente de la nada en cantidades arbitrarias para crear materia en la forma de partículas y antipartículas virtuales (incluyendo fotones virtuales) cuyos periodos de vida $(\Delta t)$ serán pequeños si la cantidad de energía $(\Delta \mathrm{E})$ que ingresa al universo es grande, o recíprocamente, los intervalos $\Delta \mathrm{t}$ serán grandes si la cantidad $\Delta \mathrm{E}$ es pequeña. Del razonamiento anterior se deduce que cada electrón de un átomo debe estar rodeado de una capa de partículas virtuales las cuales pueden ser absorbidas o emitidas por los propios electrones del átomo en un intervalo de tiempo $\Delta \mathrm{t}$ arbitrario. Por ejemplo, supongamos que la Fig.3 simule a una capa de partículas virtuales que rodean a un electrón de un átomo. Si e es la carga eléctrica del electrón con radio vector $\vec{r}_{e}$ y $\rho(\vec{r})=e \delta\left(\vec{r}-\vec{r}_{e}\right)$ su densidad de carga en cualquier punto $\overrightarrow{\mathrm{r}}$ del espacio que lo rodea (donde $\delta$ es la función de Dirac), entonces la energía de interacción del electrón con su capa (llamada "autoenergía") se expresa por:

$$
E=\int \frac{\mathrm{e}^{2} \delta\left(\overrightarrow{\mathbf{r}}-\overrightarrow{\mathbf{r}}_{\mathrm{e}}\right) \mathrm{d}^{3} \mathrm{r}}{\left|\overrightarrow{\mathbf{r}}-\overrightarrow{\mathbf{r}}_{\mathrm{e}}\right|}
$$

En la Fig.3, y de la Ec. (6) es evidente que a medida que la distancia $\left|\vec{r}-\vec{r}_{e}\right|$ al electrón disminuye, la energía $\mathrm{E}$ de su capa de partículas virtuales aumenta hacia el infinito. Cuando $\vec{r}=\vec{r}_{e}$, la Ec.(6) da:

$$
\mathrm{E}=\frac{\mathrm{e}^{2}}{0}=\infty \quad \text { (Energía infinita) }
$$

Pero según la ecuación de Einstein: $E=m_{e} c^{2}$, entonces se deduce que $\mathrm{m}_{\mathrm{e}}=\infty$. En 
consecuencia, nuevamente se llega a la conclusión absurda de que el electrón sería infinitamente "pesado".

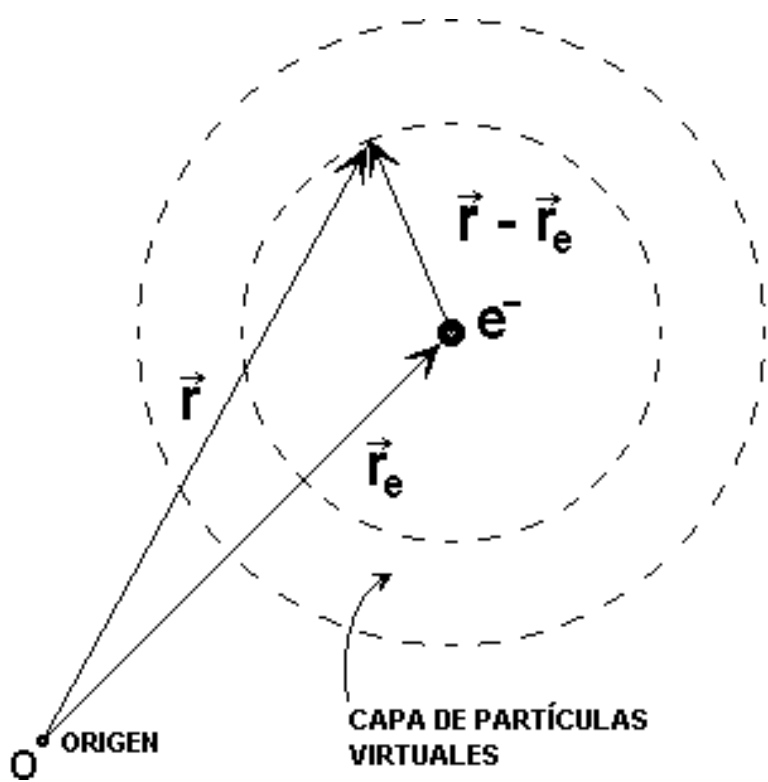

Fig. 3. Si un electrón está revestido de partículas virtuales, al interactuar con su capa de partículas virtuales adquirirá energía infinita.

Observemos que este desconcertante resultado proviene de aplicar el principio de incertidumbre (5). Esto significa que el electrón no puede ser reconocido como una partícula material puntual. Sin embargo en el experimento de Thomson la masa del electrón fue medida con la suposición errónea de que el electrón era una partícula material que obedecía a las leyes clásicas de la mecánica y del electromagnetismo. ¡Es evidente que tenemos aquí una grave contradicción!. En consecuencia los resultados del experimento de Thomson serían engañosos por principio. Además, recordemos que a partir del experimento de Thomson se desarrollaron las teorías atómica, nuclear, molecular y de partículas elementales. Entonces llegamos a la conclusión de que todos los aparatos y experimentos diseñados con la idea equivocada de que los electrones y demás partículas subatómicas son partículas materiales son dudosos por principio. El fracaso del modelo de partícula puntual fue una señal de alarma dirigida a los físicos teóricos para que renuncien definitivamente a identificar a las partículas subatómicas como objetos materiales. Sin embargo, Louis De Broglie inventó que las partículas subatómicas debían tener doble "personalidad": se comportan como objetos materiales e inmateriales, llamados ondas (principio de dualidad).

Esta propuesta salvó la crisis de la teoría atómica de la materia, la cual consistía en que las leyes de la física clásica predecían que el electrón en el átomo de hidrógeno debía "caer" hacia el núcleo atómico siguiendo una trayectoria en espiral porque radiaba energía electromagnética sin límite produciéndose un colapso y liberando una cantidad infinita de energía en un intervalo de tiempo finito, lo cual significaba que los átomos deberían ser inestables. La mecánica cuántica, que fue desarrollada por W. Heisenberg, E. Schrödinger, P. Dirac y otros, no describe a los electrones de los átomos como partículas puntuales sino como superposición de ondas, que puede interpretarse como una distribución de probabilidad alrededor del núcleo. En esta teoría se evita el colapso de los átomos porque se abandona el determinismo estricto de la mecánica clásica, reemplazándolo por una interpretación probabilística de los acontecimientos. En otras palabras, se pierde nuestra noción intuitiva de ley física a escala microscópica, y se le reemplaza por el concepto estadístico-matemático de probabilidad. Sin embargo, ¡los resultados obtenidos por todas las teorías físicas contemporáneas construidas sobre los cimientos de la mecánica cuántica, de la relatividad especial y general han fracasado en su intento de explicar cómo funciona la naturaleza porque solo nos muestran que el funcionamiento de la naturaleza es complejo!

\section{Conclusiones}

El modelo de partícula fundamental como una esfera indeformable e indestructible viola uno de los principios de la relatividad, porque ninguna interacción entre estos objetos puede transmitirse instantáneamente. En consecuencia, se pueden deformar, e incluso, desintegrarse. En el modelo de partícula elemental puntual, la combinación de la ley de Coulomb (1) y la ecuación de Einstein (3) conduce al resultado absurdo de que las partículas subatómicas serían infinitamente "pesadas". El principio de incertidumbre de la mecánica cuántica predice la creación de partículas y antipartículas virtuales (en las vastas regiones de vacío aparente de los átomos) a costa 
de la violación temporal de la ley de conservación de la energía. Pero si se usa el modelo de partícula elemental puntual para el electrón, revestido de partículas y antipartículas virtuales, este resulta ser infinitamente "pesado" El concepto de partícula fundamental no puede sostenerse ante estos resultados absurdos. ¡Esto ha significado un retraso hacia la verdadera unificación de la física con la que, las futuras generaciones de seres humanos adquirirían gradualmente una nueva y significativa visión del paraíso natural que observamos!

\section{Referencias}

[1] Weinberg, S. (1985). Partículas Subatómicas. Prensa Científica, S.A., Barcelona, 1985.

[2] Hawking, S. W. (1989). Historia del Tiempo. Del Big Bang a los agujeros negros. Ed. Retina, Bogotá.

[3] Bondi, H. (1970). Cosmología. Editorial Labor, S.A. Barcelona.
[4] Pais, A. (1979). "Einstein and the quantum theory". Reviews of Modern Physics, 51, 863.

[5] Bernstein, J. y Gerald Feinberg, Editores. (1986). Cosmological Constants. Papers in Modern Cosmology, New York.

[6] Lederman, L. M. (1984). The Value of Fundamental Science. Sci. Am., Noviembre.

[7] Quigg, C. (1985). Elementary Particles and Forces. Sci. Am., Abril.

[8] Weinberg, S. (1983). The Discovery of Elementary Particles, Scientific American Library, New York, W.N. Freeman Company.

[9] Fritzsch, H. (1983). Quarks, The Stuff of Mater, London, Allen and Lang.

[10] Harari, H. (1983). The Structure of Quarks and Leptons. Sci. Am., Abril. 